
\title{
3 Research Square \\ Deltex1 promotes cell proliferation and invasion and associated with clinical characteristics of breast cancer
}

\section{Xiaoyi Liu}

the Affiliated Hospital of Qingdao University

Jianxia Hu

the Affiliated Hospital of Qingdao University

Kui Che

the Affiliated Hospital of Qingdao University

Jingwei Chi

Qingdao Women and Childrens Hospital

\section{Zhengju Fu}

the Affiliated Hospital of Qingdao University

Xiangping Liu

the Affiliated Hospital of Qingdao University

haibo wang ( $\nabla$ qdyxywhb@163.com )

the Affiliated Hospital of Qingdao University https://orcid.org/0000-0001-6545-495X

\section{Research article}

Keywords: Deltex1, Breast cancer, PI3K/AKT signal, Notch pathway

Posted Date: March 26th, 2020

DOI: https://doi.org/10.21203/rs.3.rs-19340/v1

License: (c) (1) This work is licensed under a Creative Commons Attribution 4.0 International License. Read Full License 


\section{Abstract}

\section{Background}

Deltex1 (DTX1) was found to play its role in the development of several kinds of cancer. The association between DTX1 and breast cancer was unclear. We aimed to investigate the association between DTX1 expression and clinical characteristics of breast cancer, further explore the possible mechanisms of DTX1 on the development of breast cancer.

\section{Methods}

We determined level of DTX1 expression by qRT-PCR, analyzed association between DTX1 and clinical characteristics of breast cancer by student's test and ANOVA test. The possible mechanisms of DTX1 on the development of breast cancer were explored with breast cancer cell lines in vitro.

\section{Results}

Levels of DTX1 in breast cancer tissues were lower compared to FCD tissues and peri-neoplastic breast tissues $(P<0.01)$. Lower expression of DTX1 was associated with advanced tumor grade (grade III, $P=0.002)$, advanced clinical TNM stage (III-IV stage, $P=0.017)$, positive lymph node metastasis $(P=0.034)$ and high $\mathrm{Ki}-67$ index $(P=0.031)$. Multivariate Cox regression analysis revealed that $D T X 1$ expression was recognized as an independent prognostic factor for MFS in breast cancer. Elevated levels of Notch1, Jagged 1 and HES1 in NOTCH pathway and p-Akt/PKB in PI3K/AKT pathway were found in breast cancer cells with lower DTX1 expression. GSI treatment could inhibit breast cancer cell proliferation and migration through NOTCH/DTX1/PI3K/AKT pathways.

\section{Conclusion}

Lower DTX1 in breast tissues was associated with advanced condition, prognosis and metastasis of breast cancer. Lower DTX1 might promote breast cancer cell proliferation and migration via NOTCH/DTX1/PI3K/AKT pathways. DTX1 might be useful as a marker to select new therapy for breast cancer.

\section{Background}

Breast cancer is the most common malignancy in women. Due to the expansion of novel therapeutic schemes and greater potential of recovery, breast cancer is considered as a better researched cancer in the past twenty years [1]. Current treatment approach to breast cancer is individualized and multimodal, surgical treatment is essential in therapeutic approach. However, several main issues such as disease recurrence and distant metastases still persist [2]. It is urgent to investigate and find new biomarker and target to explore better drug or therapy for breast cancer. 
Deltex 1 (DTX1) is a single transmembrane protein with ubiquitin E3 ligase activity, also a downstream molecule and regulator of Notch signal pathway in mammals [3]. DTX1 over-expression inhibited Notch signaling, as demonstrated by enhanced B cell lymphocyte development and suppressed T cell development [4, 5]. DTX1 was induced in T-cell anergy and could inhibit T-cell activation in E3-dependent and E3-independent mechanisms [4]. DTX1 promoted B cell differentiation through specific enhancement of E47 activity [5]. However, its exact role in mammals still needs further investigations.

Recently, DTX1 was found to play its role in the development of several kinds of cancer. DTX1 was downregulated in gastric cancer tissues, and linked to better prognosis in gastric cancer [3]. DTX1 mutations were identified in Chinese patients with primary and relapsed diffuse large B-cell lymphoma [6]. Glioblastoma (GBM) patients with low DTX1 levels had a more favorable prognosis, Notch pathway via DTX1 appeared to be an oncogenic factor in glioblastoma [7]. DTX1 regulated osteosarcoma invasiveness through Notch/HES1, low DTX1 expression might be useful as a marker to select osteosarcoma patients who could benefit from Notch inhibitor treatment [8]. However, there was little report about association between DTX1 and breast cancer.

Notch pathway is a highly conserved cell signaling pathway that plays a key role in many cellular processes, including proliferation, survival and apoptosis [9]. Notch signaling function in cancer may be oncogenic in some kinds of cancer [10-13]. Notch1 overexpression has been reported to promote tumor growth in various cancers like myeloma [10], breast cancer [11], cervical cancer [12] and hepatocellular carcinoma [13], etc. Notch1 gene silencing in gastric and prostate cancer cells inhibited tumor growth [14, 15]. Moreover, it was reported that Notch1, HES1, and DTX1 genes could be considered as potential biomarkers for GBM diagnosis [7].

In the present research, we investigated the association between DTX1 expression and breast cancer, and the relationship of DTX1 and clinical characteristics of breast cancer, further explored the possible mechanisms of DTX1, Notch signaling passway on the development of breast cancer.

\section{Methods}

\section{Patients and breast cancer specimens}

This study was approved by the institutional review board in the Affiliated Hospital of Qingdao University. The experiments were undertaken with the understanding and written informed consent of each subject before enrollment in this study. The study conformed with the Code of Ethics of the World Medical Association (Declaration of Helsinki).

All of patients and healthy controls were enrolled in the study from the same geographic area (Qingdao, Shandong) from March 2015 to February 2017. All patients with breast cancer or fibrocystic dysplasia (FCD) were diagnosed according to their clinical and pathologic manifestation, as defined by WHO classification criteria [16]. The patient was considered hormone receptor positive according to the assay results of primary tumor tissue acquired by biopsy. Main exclusion criteria were inflammatory breast 
cancer; other concurrent or previous malignant disease; life-threatening disease, such as uncontrolled cardiac diseases; or a pregnant or lactating status; radio or chemotherapy administration before surgery. The healthy controls were from people who had physical examination in our hospital, with age and gender matched.

\section{Histopathological examination}

Surgical specimens or tumor tissues acquired by biopsy were microscopically examined by two or more experienced pathologists, following histopathologic factors were assessed: cell type of the main lesion, primary tumor size, location, multiplicity, bilaterality, margin involvement, lymph-vascular invasion and lymph node metastasis (LNM).

\section{RNA extraction and RT-qPCR}

Total RNA from cell lines, breast tissues and peripheral blood was extracted using RNeasy Mini Kit (Qiagen). The concentration and quantity of total RNA were determined based on the absorbance at $260 \mathrm{~nm}$ using a NANO DROP spectro- photometer (ThermoScientific, USA).

PCR was conducted in $25 \mu$ reaction volume, containing $12.5 \mu$ l Maxima SYBR Green qPCR Master Mix (2X) and $300 \mathrm{nM}$ of each primers. The primers sequences were as follows: DTX1, forward primer: 5'GGGCTGATGCCTGTGA ATG-3', reverse primer: 5'-CCTGGCGAAACTGGTGC-3'. A pre-incubation at $95^{\circ} \mathrm{C}$ for 10 min was to activate the Hot Start DNA polymerase and denature DNA, and was followed by 45 amplification cycles of $95^{\circ} \mathrm{C}$ denaturation for $10 \mathrm{sec}, 60^{\circ} \mathrm{C}$ annealing for $20 \mathrm{sec}$. qRT-PCR was performed in ABI PRISM 7900 Realtime PCR system (Applied Biosystems).

\section{Cell culture and Transfection}

The human breast cancer cell lines, HCC1937 and BT474, were obtained from American type culture collection (ATCC, Manassas, VA, USA). Each cell line was authenticated using a Short Tandem Repeat (STR) Identifiler kit (Applied Biosystems). HCC1937 and BT474 cells were grown on Dulbecco's modified Eagle's medium (DMEM; Thermo Fisher Scientific) with 10\% FBS (HyClone, Logan, UT, USA) at $37^{\circ} \mathrm{C}$ in $5 \%$ $\mathrm{CO}_{2}$.

For knockdown assays, the expression of DTX1 gene was downregulated by ON-TARGET plus siRNA SMART pool RNA (Thermo Scientific, Waltham, MA) using RNAi MAX transfection reagent (Life Technologies, Carlsbad, CA). Non-targeting SMART pool RNA (Life Technologies) was used as a control. The transfection efficiency was confirmed by qRT-PCR.

The ectopic overexpression of DTX1 was achieved with pCMV6-Entry-DTX1 plasmid (RC208338, Origene, Rockville, MD) using FuGENE Extreme 9 transfection reagent (Roche, Nutley, NJ). Empty pCMV6-Entry (PS100001, Origene) was used as a control. Transfection efficiency was confirmed by qRT-PCR. 
Drug solutions were prepared fresh before each dose using a dounce homogenizer. For GSI (GSI-IX, MedchemExpress) treatment in vitro experiments, stocks were prepared at $10 \mathrm{mM}$ in DMSO and dilutions were made directly before use. Cells were cultured for 3 days with GSI (compound E, 10uM) to establish a Notch-off state.

\section{Cell proliferation analysis}

Cell proliferation was analyzed using the 'Amersham Cell Proliferation Biotrak ELISA, version 2' system (GE Helathcare, UK) according to manufacturer's instructions. In short, 5'000 cells were seeded in the well of a 96-well plate and grown for two days, labeled with BrdU for 3-4 h, fixed and labeled with a peroxidaselabeled anti-BrdU antibody. After coloring reaction the optical density was measured with a 'SpectraMAX 250' plate reader and analyzed with accompanying 'Soft Max Pro' software (Molecular Devices, MDS Analytical Technologies, Toronto, Canada). For cell counting, equal amounts of cells were seeded in triplicates and grown under standard conditions for three days. Cells were then harvested and each biological replicate was counted 3 times using a 'Neubauer'-chamber (hemacytometer).

\section{Transwell migration and scratch test assay}

Transwell migration assays were performed using modified Boyden chamber units with polycarbonate filters of $8 \mathrm{~mm}$ porosity (Costar, Vitaris, Switzerland). The lower side of the filter was coated with $25 \mathrm{mg} / \mathrm{ml}$ collagen 1 (Sigma, St. Louis, USA) for $2 \mathrm{~h}$ at $37^{\circ} \mathrm{C}$. The bottom chamber was filled with DMEM containing $10 \%$ FCS. Cells $\left(2 \searrow 10^{4}\right.$ per well in serum-free DMEM) were plated in the upper chamber in $100 \mathrm{ml}$ medium and incubated for $24 \mathrm{~h}$ in standard conditions (see above). After removal of the remaining cells from the upper surface of the filter insert, migrated cells at the bottom of the filter were fixed with $3.7 \%$ formaldehyde in PBS and stained with $0.1 \%$ crystal violet. For every individual filter, the cells in 9 fields of view were counted. Every experiment was conducted in triplicates.

For scratch test analysis, cells were grown to above $90 \%$ confluency under standard conditions. A wound was inflicted by scratching a $200 \mathrm{ml}$ pipette tip (Starlab, Milton Keynes, UK) over the surface of the culture flask. The wounds were documented immediately after scratching, after 12, 24 and 48 hours. Quantification of wound closing was performed with ImageJ software according to manufacturers' instructions.

\section{Western blot analysis and antibodies}

Protein lysates from tissues or cells were resolved on denaturing 8-12\% SDS-poly-acrylamide gels and transferred to nitrocellulose membranes (iBlot Gel transfer stacks, Invitrogen). The following primary antibodies were used: anti-Actin (Sigma-Aldrich, St. Louis, USA), anti-phospho-Akt/PKB and anti-totalAkt/PKB (Ser-473) (Millipore), anti-DTX1 (AB Biotech). Decorated proteins were revealed using horseradish peroxidase-conjugated anti-mouse, anti-rabbit, anti-rat (New England Biolabs) secondary antibodies and visualized by the chemoluminescence detection system Super-Signal West Pico (Thermo 
Scientific). Densitometry of western blots was performed using Image $\mathrm{J}$ software according to manufacturers' instructions.

\section{Statistical analysis}

SPSS 19.0 was used for statistical analysis. Data were presented as the mean \pm standard deviation (SD). Student's paired or unpaired t test was used to analyze significance between paired or unpaired groups. One-way analysis of variance (ANOVA) test was used to analyze significance between groups of various differentiation. Only significant results $\mathrm{P}<0.05$ after Bonferroni correction were the focus of this report.

\section{Results}

1. Patients and clinical characteristics

We enrolled 463 patients with breast cancer, 120 patients with FCD and 120 healthy controls in the present study. The mean age in healthy controls and patients with breast cancer were $46.9 \pm 11.5 \mathrm{y}$ (range $23-65 \mathrm{y})$ and $50.3 \pm 9.7 \mathrm{y}(18-65 \mathrm{y})$, while the mean age in patients with FCD was $40.4 \pm 14.3 \mathrm{y}(19-43$ y) for the younger peak age of this disease. Breast cancer patients were staged according to the TNM classification. There were 102 patients with stage I (22.03\%), 175 patients with stage II (37.8\%), 140 patients with stage III (30.24\%) and 46 patients with stage IV $(9.94 \%)$. All the clinical characteristics were shown in Table 1.

\section{DTX1 is expressed in breast tissues}

We determined mRNA expression of DTX1 in serum and breast tissues of all subjects with qRT-PCR to confirm its presence in breast cancer. As showed in Fig. 1, no significant differences were found in serum levels of DTX1 mRNA expression between controls, patients with FCD and patients with breast cancer. While in breast tissues, mRNA expression levels of DTX1 in breast cancer were lower compared with FCD tissues and perineoplastic breast tissues $(P<0.01)$. We further analyzed the expression of DTX1 between the four subtypes of breast cancer, no significant difference was found.

\section{Associations between DTX1 and clinical characteristics of breast cancer}

We further analyzed the associations between levels of DTX1 and clinical characteristics of breast cancer. As shown in Table 2, level of DTX1 was not obviously associated with age, body mass index (BMI), pathology type, hormone receptor (HR) status, HER2 status, or tumor size in patients with breast cancer, while lower expression of DTX1 was associated with advanced tumor grade (grade III, $P=0.002$ ), advanced clinical TNM stage (III-IV stage, $P=0.017)$, positive lymph node metastasis $(P=0.034)$ and high Ki-67 index $(P=0.031)$. This indicated that lower DTX1 was associated with advanced condition and poor prognosis. In addition, multivariate Cox regression analysis revealed that DTX1 expression, LNM, clinical stage, HER2 status, and Ki-67 index were each recognized as independent prognostic factors for metastasis-free survival (MFS) in breast cancer (Table 3). These results demonstrated that lower DTX1 might contribute to the metastasis of breast cancer. 
4. Lower DTX1 promoted breast cancer cell proliferation, migration and invasion

The results of our clinical samples demonstrated that lower DTX1 expression might be associated with cancer cell proliferation, invasion and metastasis in breast cancer. Then we explored the possible mechanisms of DTX1 on breast cancer. The DTX1 expression in different cell lines were shown in Fig. 2A. HCC1937 and BT474 cell lines were chosen for DTX1 knockdown and overexpression studies (Fig. 2B). The growth of HCC1937/shDTX1 cells was $141 \%$ of the rate of control cells while BT474/DTX1 cells grew in monolayer culture at $62 \%$ of the rate of control cells $(p<0.01$, as shown in Fig. $2 C)$.

To determine the role of DTX1 on invasion and metastasis of breast cancer cell, we performed transmembrane invasion assays with HCC1937/shDTX1, BT474/DTX1 cell and control cells. Lowexpression of DTX1 significantly promoted the invasive behavior of HCC1937 cells by $154 \%$ of control cells, while BT474/DTX1cells showed an average reduction of invasion by $65 \%$ of control cells $(P<0.01$, Fig. 2C).

To confirm these results in an independent experimental setup, we performed scratch test wound healing assays. Wounds were imaged immediately after the scratch wound was inflicted as well as 0,24 and 48 hours later. Wound closing, which is a measure for cell motility, was massively accelerated in HCC1937/shDTX1 cells compared to controls ( $p<0.01$, Fig. 2D) whereas wound closing was significantly reduced in BT474/DTX1 cells ( $p<0.05$, Fig. 2E). It confirmed the initial finding of the trans-well migration and invasion assay.

5. DTX1 regulated cell growth and survival via NOTCH/DTX1/ PI3K/AKT pathways

Since DTX1 is a regulator of the Notch pathway, and PI3K/AKT pathway is known to involve in cell proliferation in breast cancer, we analyzed associations between DTX1, Notch pathway and PI3K/AKT pathway in breast cancer cells.

In BT474/DTX1 cells, we observed a reduction of the phospho-Akt/PKB (p-Akt/PKB) levels compared to BT474/scr cells. In contrast, we detected a slight increase of p-Akt/PKB levels in the HCC1937/ shDTX1 cells compared to HCC1937/scr cells (as shown in Fig. 3A). This was consistent with the results of cell growth. Expressions of Notch1, Delta-like 1 (DLL1), Jagged1 and HES1 of Notch signaling pathway were significantly upregulated in HCC1937/ shDTX1 cells relative to HCC1937/scr cells by qRT-PCR $(P<0.01$, as shown in Fig. 3B). While expressions of these molecules were decreased in BT474/DTX1 cells compared to BT474/scr cells $(P<0.01)$. These findings indicated that DTX1 might be a key factor to regulate the activation of Notch signal pathway and PI3K/AKT pathway in breast cancer.

To further confirm that DTX1 regulated cell growth and migration via NOTCH/DTX1/ PI3K/AKT pathways, we treated BT474 cells with Gamma-secretase inhibitor (GSI), a Notch blocker. After GSI treatment, the proliferation and migration of BT474 cells decreased by $38 \%$ and $55 \%$ of controls $(P<0.01$, Fig. 4). The wound closing of BT474 cells with GSI reduced significantly compared to BT474 cells without GSI. Moreover, expression of DTX1 increased while p-Akt/PKB levels decreased in BT474 cells 
with GSI. These indicated that GSI could inhibit BT474 cell proliferation and migration through NOTCH/ DTX1/PI3K/AKT pathways.

\section{Discussion}

The role of DTX1 in oncogenesis is not clearly understood. It was reported that Notch1, HES1 and DTX1 could be considered as potential biomarkers for Glioblastoma diagnosis [7]. In the present study, we explored the associations between DTX1 and breast cancer, and found that levels of DTX1 in breast cancer tissues were lower compared to FCD tissues and perineoplastic breast tissues. Lower DTX1 was associated with advanced condition and poor prognosis of breast cancer.

Expression of DTX1 gene is not only present in a variety of tissues such as lymphoid tissues, heart, liver and kidney [17], but also in tumors with a centroblast expression profile [18]. DTX1 played various roles in different types of cancers. Over-expression of DTX1 increased cell migration and invasion correlating to ERK activation, miR-21 and endogenous Notch levels in glioblastoma [7]. While downregulated DTX1 maybe associated with Notch pathway activation and increased migration potential in Head and Neck Squamous Cell Carcinoma [19]. In our experiment, lower expression of DTX1 was associated with advanced tumor grade, advanced clinical TNM stage, positive lymph node metastasis and high Ki-67 index in breast cancer. Also, our results revealed that lower DTX1 expression might be an independent prognostic factor for MFS in breast cancer.

Unlimited replicative potential of tumor cells is a hallmark of aggressive cancers [20]. Elevated levels of pAkt/PKB have been shown to positively correlate with migrating breast cancer cells [21]. Hyperactivation of PI3K/AKT pathway is involved in the progression of breast cancer and contributes to the poor outcome [22]. Our results found that lower DTX1 expression was associated with higher cancer cell proliferation and increased invasion and metastasis, and moreover these findings were related with PI3K/AKT pathway. PI3K/AKT pathway in BT474 cells with lower DTX1 expression was hyper-activated while inhibited in BT474/DTX1 with higher DTX1 expression cells. This was consistent with the results of breast cancer cell growth. A direct link between DTX1 and PI3K/AKT pathways has so far not been described, we aimed to identify molecular mediators involved in the further investigations.

Notch signaling pathway participates in neoplastic transformation in various cell types, such as cervical cancer, lung cancer and breast cancers. High expressions of Notch-1 and its ligand Jagged-1 were associated with poor prognosis in breast cancer [23]. Notch-1, DLL1 and Jagged-1 have been found to be upregulated in clear cell renal cell carcinoma and lung adenocarcinoma in vitro and in vivo models [2426]. Notch inhibition resulted in decreased proliferation and self-renewal of Glioblastoma cells [27]. However, the mechanisms of Notch signaling in cancers promotes cell invasion and metastasis were not fully understood.

The Notch pathway is usually determined by other signaling pathways which cross talk with it, including NF-KB, PI3K, AKT and mTOR [28]. PI3K/AKT pathway has been reported to be involved with NF-KB activation in a wild variety of tumors [29-31]. Activated Notch-1 might induce the activation of PI3K/AKT 
pathway, then upregulated NF-KB activity to facilitate cell invasion [32]. More work is needed to further understand factors that promote AKT phosphorylation in the context of Notch-1 activation.

DTX1 is the downstream molecule of Notch signaling pathway and also the critical regulator in Notch signaling pathway [33]. In the present research, Notch1, HES1, DLL1 and Jagged1 in Notch pathway were significantly upregulated in cells with lower DTX1 expression compared with DTX1 high expression cells. These findings confirmed that downregulation of DTX 1 resulted in a strong difference in activation of the NOTCH pathway in breast cancer samples. GSI could inhibit the proliferation and migration of BT474 cell, inducing increased DTX1 and decreased p-Akt/PKB. Based on the above findings, DTX1 might be a key factor to regulate the activation of Notch signal pathway and PI3K/AKT pathway in breast cancer. The specific mechanisms of DTX1 regulated invasion and metastasis via Notch/DTX1/ PI3K/AKT pathways still need further investigations.

There were some limitations in the present research. First, the specific mechanisms of DTX1 promoted breast cancer cell proliferation and invasion were not fully understood. Second, how DTX1 activated the $\mathrm{PI} 3 \mathrm{~K} / \mathrm{AKT}$ pathway and regulated Notch/DTX1/PI3K/AKT pathways still needed further investigations. It is a long way to make clear the role and mechanisms of DTX1 in breast cancer and further to find new target drug to evaluate in clinical trials.

In conclusion, we found that lower DTX1 in breast cancer tissues was associated with advanced condition and poor prognosis and contributed to the metastasis of breast cancer. Lower DTX1 might promote breast cancer cell proliferation and migration via NOTCH/DTX1/PI3K/AKT pathways. DTX1 may be a useful marker to select new therapy for breast cancer.

\section{Abbreviations}

DTX1

Deltex1

FCD

fibrocystic dysplasia

GBM

Glioblastoma

LNM

lymph node metastasis

MFS

metastasis-free survival

BMI

body mass index

HR

hormone receptor

ER 
estrogen receptor

PR

progesterone receptor

HER2

human epidermal growth factor receptor-2

PI3K

phosphoinosmde-3-kinase

DLL1

Delta-like 1

\section{Declarations}

\section{Ethics approval and consent to participate}

This study was approved by the Institutional Animal Ethical Committee, Qingdao and Ethics Committee of the Affiliated Hospital of Qingdao University. All applicable international, national, and/or institutional guidelines for the care and use of animals were followed

\section{Consent for publication}

Not applicable

\section{Availability of data and materials}

The datasets used and/or analysed during the current study are available from the corresponding author on reasonable request.

\section{Competing interests}

The authors declare that they have no competing interests.

\section{Funding}

Project of Youth Research Fund in the Affiliated Hospital of Qingdao University in 2018. The funder was the Affiliated Hospital of Qingdao University, our hospital provides financial support for all young outstanding researchers, hoping to achieve excellent findings and contribute to human health.

\section{Authors' contributions}

$\mathrm{JH}$ : designed the experiment, direct the whole experiment, writed, revised and proved the manuscript. 
KC and JC: performed cell culture and blood samples examination.

ZF and $\mathrm{XL}$ : performed the breast tissues and signaling pathway examination

$\mathrm{HW}$ and $\mathrm{XL}$ : directed the experiment and revised the manuscript.

\section{All authors read and approved the final manuscript.}

\section{Acknowledgments}

We appreciated all pathologists in this study.

\section{References}

1. Kast K, Link T, Friedrich K, Petzold A, Niedostatek A, Schoffer O, et al. Impact of breast cancersubtypes and patterns of metastasis on outcome.Breast Cancer Res Treat. 2015; 150(3):6219.

2. Eriksson M, Czene K, Pawitan Y, Leifland K, Darabi H, Hall P. A clinical model for identifying the shortterm risk of breast cancer.Breast CancerRes. 2017; 19(1):29.

3. Hsu TS, Mo ST, Hsu PN, Lai MZ. c-FLIP is a target of the E3 ligasedeltex1 in gastric cancer. Cell Death Dis. 2018; 9(2):135.

4. Hsu TS, Hsiao HW, Wu PJ, Liu WH, Lai MZ. Deltex1promotes protein kinase C $\theta$ degradation and sustains Casitas B-lineage lymphoma expression. J Immunol. 2014; 193 (4):1672-80.

5. Asashima $H$, Tsuboi $H$, Takahashi $H$, Hirota $T$, lizuka $M$, Kondo $Y$, et al. The anergy induction of $M 3$ muscarinic acetylcholine receptor-reactive $\mathrm{CD} 4+\mathrm{T}$ cells suppresses experimental sialadenitis-like Sjögren's syndrome.Arthritis Rheumatol. 2015; 67(8):2213-25.

6. Meriranta L, Pasanen A, Louhimo R, Cervera A, Alkodsi A, Autio M, et al. Deltex-1 mutations predict poor survival in diffuse large B-cell lymphoma.2017; 102(5):e195-8.

7. Huber RM, Rajski M, Sivasankaran B, Moncayo G, Hemmings BA, Merlo A. Deltex-1 activates mitotic signaling and proliferation and increases the clonogenic and invasive potential of U373 and LN18 glioblastoma cells and correlates with patient survival. PLoS One. 2013; 8(2):e57793.

8. Zhang P, Yang Y, Nolo R, Zweidler-McKay PA, Hughes DP. Regulation of NOTCH signaling by reciprocal inhibition of HES1 and Deltex 1 and its role in osteosarcoma invasiveness. 2010; 29(20):2916-26.

9. Bocci F, Gearhart-Serna L, Boareto M, Ribeiro M, Ben-Jacob E, Devi GR, et al. Toward understanding cancer stem cell heterogeneity in the tumor microenvironment. Proc Natl Acad Sci U S A. 2019; 116(1):148-57.

10. Saltarella I, Frassanito MA, Lamanuzzi A, Brevi A, Leone P, Desantis V, et al. Homotypic and Heterotypic Activation of the Notch Pathway in Multiple Myeloma-Enhanced Angiogenesis: A Novel 
Therapeutic Target? Neoplasia. 2019; 21(1):93-105.

11. Kumar S, Srivastav RK, Wilkes DW, Ross T, Kim S, Kowalski J, et al. Estrogen-dependent DLL1mediated Notch signaling promotes luminal breast cancer. Oncogene. 2019; 38(12):2092-107.

12. He G, Mu T, Yuan Y, Yang W, Zhang Y, Chen Q, et al. Effects of Notch Signaling Pathway in Cervical Cancer by Curcumin Mediated Photodynamic Therapy and Its Possible Mechanisms in Vitro and in Vivo. J Cancer. 2019; 10(17):4114-22.

13. Xiong S, Wang R, Chen Q, Luo J, Wang J, Zhao Z, et al. Cancer-associated fibroblasts promote stem cell-like properties of hepatocellular carcinoma cells through IL-6/STAT3/ Notch signaling.Am J Cancer Res. 2018; 8(2):302-16.

14. Hibdon ES, Razumilava N, Keeley TM, Wong G, Solanki S, Shah YM, et al. Notch and mTOR Signaling Pathways Promote Human Gastric Cancer Cell Proliferation. Neoplasia. 2019; 21(7):702-12.

15. Zhang J, Kuang Y, Wang Y, Xu Q, Ren Q.Notch-4 silencing inhibits prostate cancer growth and EMT via the NF-кB pathway.Apoptosis. 2017; 22(6):877-84.

16. Ott G, Klapper W, Feller AC, Hansmann ML, Möller P, Stein H, et al. Revised Version of the 4th Edition of the WHO Classification of Malignant Lymphomas: What Is New? Pathologe, 2019; 40 (2): $157-68$.

17. Song Y, Kumar V, Wei HX, Qiu J, Stanley P. Lunatic, Manic, and Radical Fringe Each Promote T and B Cell Development. J Immunol. 2016;196(1):232-43.

18. Gupta-Rossi N1, Storck S, Griebel PJ, Reynaud CA, Weill JC, Dahan A. Specific over-expression of deltex and a new Kelch-like protein in human germinal center B cells. Mol Immunol.2003; 39(13):7919.

19. Gaykalova DA, Zizkova V, Guo T, Tiscareno I, Wei Y, Vatapalli R, et al. Integrative computational analysis of transcriptional and epigenetic alterations implicates DTX1 as a putative tumor suppressor gene in HNSCC. Oncotarget. 2017; 8(9):15349-63.

20. Dudani JS, Ibrahim M, Kirkpatrick J, Warren AD, Bhatia SN. Classification of prostate cancer using a protease activity nanosensor library. Proc Natl Acad Sci U S A. 2018;115(36):8954-9.

21. Wang SW, Cheung HP, Tong Y, Lu J, Ng TB, Zhang YB, et al. Steroidogenic effect of Erxian decoction for relieving menopause via the p-Akt/PKB pathway in vitro and in vivo. J Ethnopharmacol. 2017; 195:188-95.

22. Sharma V, Sharma AK, Punj V, Priya P. Recent nanotechnological interventions targeting PI3K/Akt/mTOR pathway: A focus on breast cancer. Semin Cancer Biol. 2019; 59:133-46.

23. Andrieu G, Tran AH, Strissel KJ, Denis GV. BRD4 Regulates Breast cancer Dissemition through Jagged1/Notch1 Signaling. Cancer Res. 2016; 76(22):6555-67.

24. Narayanappa R, Rout P, Aithal MG, Chand AK. Aberrant expression of Notch1, HES1, and DTX1 genes in glioblastoma formalin-fixed paraffin-embedded tissues. Tumour Biol. 2016; 7(5):6935-42.

25. Ai Q, Ma X, Huang Q, Liu S, Shi T, Zhang C, et al. High-level expression of Notch1 increased the risk of metastasis in T1 stage clear cell renal cell carcinoma. PLoS One. 2012; 7(4):e35022. 
26. Donnem T, Andersen S, Al-Shibli K, Al-Saad S, Busund LT, Bremnes RM. Prognostic impact of Notch ligands and receptors in nonsmall cell lung cancer: coexpression of Notch-1 and vascular endothelial growth factor-A predicts poor survival. Cancer. 2010; 116(24):5676-85.

27. Saito N, Hirai N, Aoki K, Suzuki R, Fujita S, Nakayama H, et al.The Oncogene Addiction Switch from NOTCH to PI3K Requires Simultaneous Targeting of NOTCH and PI3K Pathway Inhibition in Glioblastoma. Cancers (Basel). 2019; 11(1).

28. Hibdon ES, Razumilava N, Keeley TM, Wong G, Solanki S, Shah YM, et al. Notch and mTOR Signaling Pathways Promote Human Gastric Cancer Cell Proliferation. Neoplasia. 2019; 21(7):702-12.

29. Zhou B, Wang D, Sun G, Mei F, Cui Y, Xu H. Effect of miR-21 on Apoptosis in Lung Cancer Cell Through Inhibiting the PI3K/ Akt/NF-KB Signaling Pathway in Vitro and in Vivo. Cell Physiol Biochem. 2018; 46(3):999-1008.

30. Torrealba N, Vera R, Fraile B, Martínez-Onsurbe P, Paniagua R, Royuela M. TGF$\beta / P I 3 K / A K T / m T O R / N F-k B$ pathway. Clinicopathological features in prostate cancer. Aging Male. 2019; 11:1-11.

31. Shao N, Lu Z, Zhang Y, Wang M, Li W, Hu Z, et al. Interleukin-8 upregulates integrin $\beta 3$ expression and promotes estrogen receptor-negative breast cancer cell invasion by activating the PI3K/Akt/NF-KB pathway. Cancer Lett. 2015; 364(2):165-72.

32. Han R, Gu S, Zhang Y, Luo A, Jing X, Zhao L, et al. Estrogen promotes progression of hormonedependent breast cancer through CCL2-CCR2 axis by upregulation of Twist via PI3K/AKT/NF-KB signaling. Sci Rep. 2018; 8(1):9575.

33. Cheng YC, Huang YC, Yeh TH, Shih HY, Lin CY, Lin SJ, et al. Deltex1 is inhibited by the NotchHairy/E(Spl) signaling pathway and induces neuronal and glial differentiation. Neural Dev. $2015 ; 10: 28$.

\section{Tables}

Due to technical limitations, Tables 1-3 are provided in the Supplementary Files section.

\section{Figures}


A

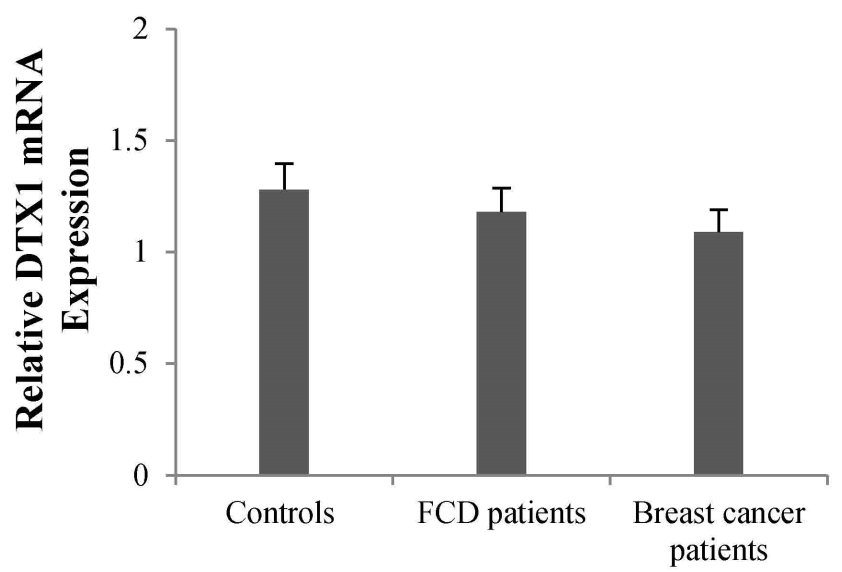

B

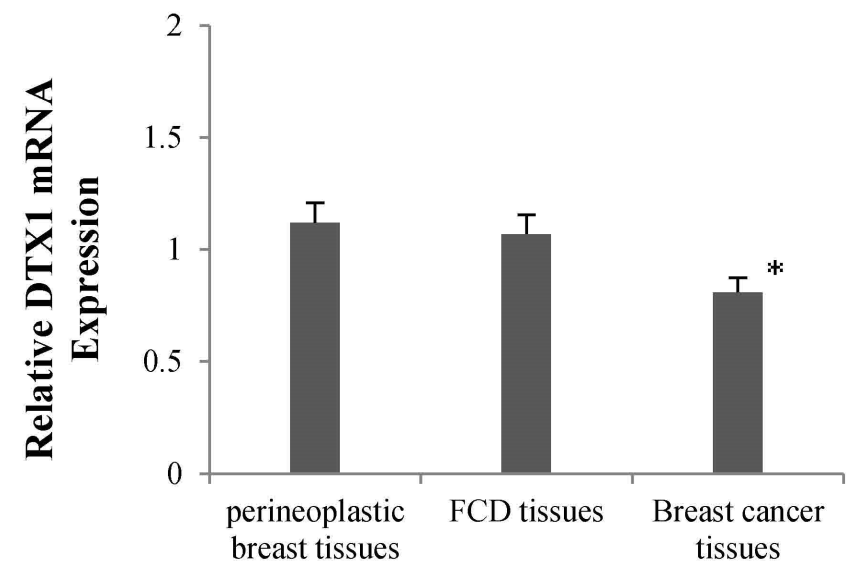

\section{Figure 1}

DTX1 expression in breast tissues. A: No significant differences were found in serum levels of DTX1 mRNA expression between controls, FCD patients and breast cancer patients. B: mRNA expression level of DTX1 in breast cancer tissues was lower compared to FCD tissues and perineoplastic breast tissues (* $\mathrm{P}<0.01)$. 
A

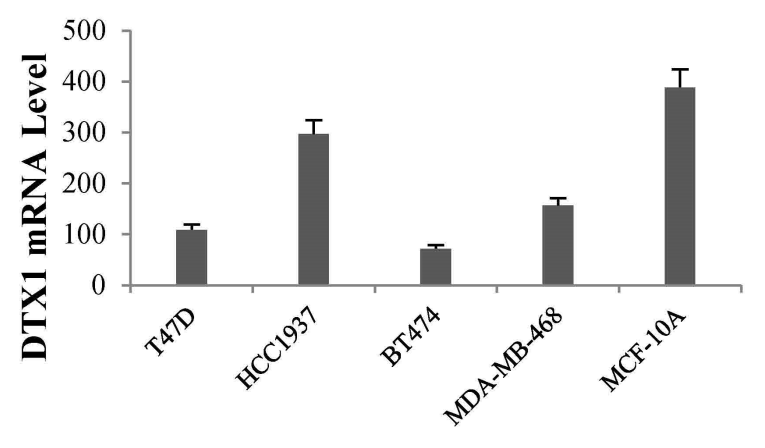

C
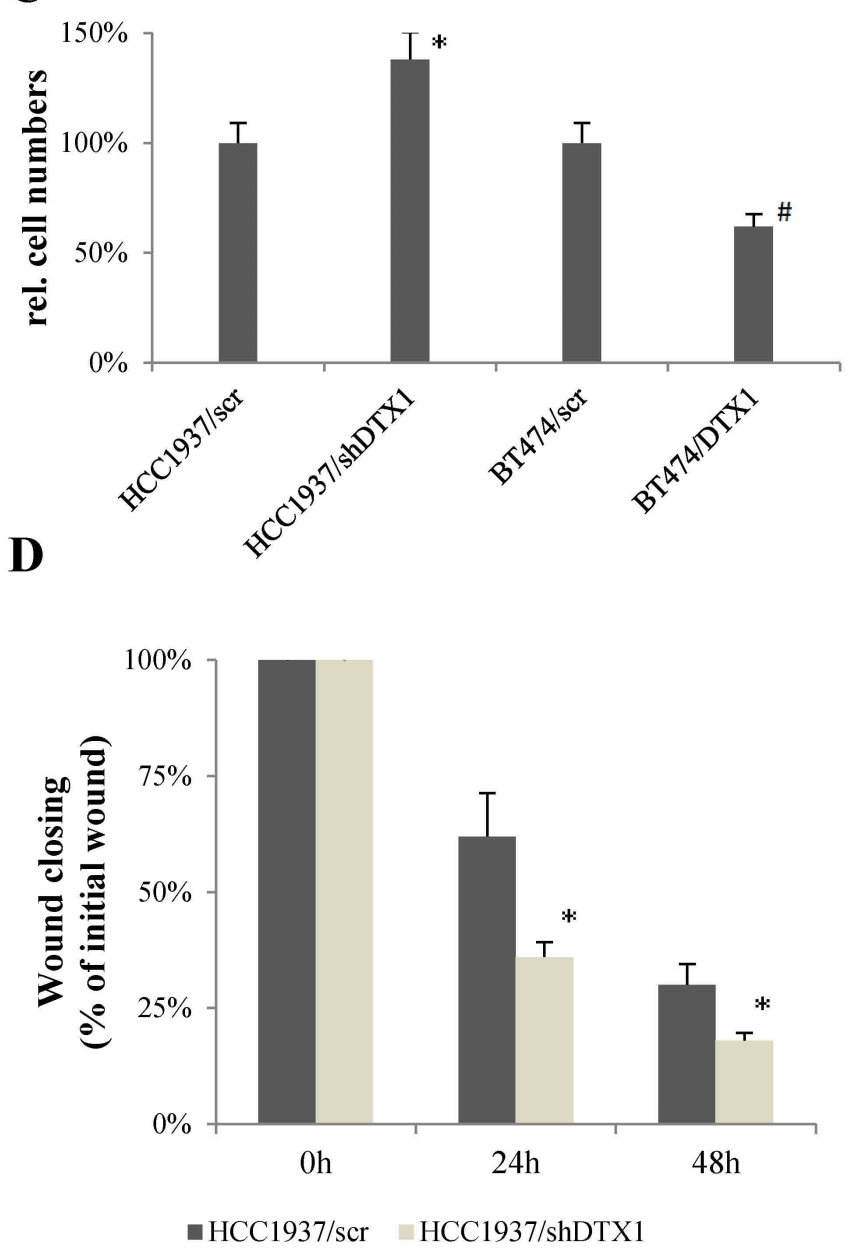

B

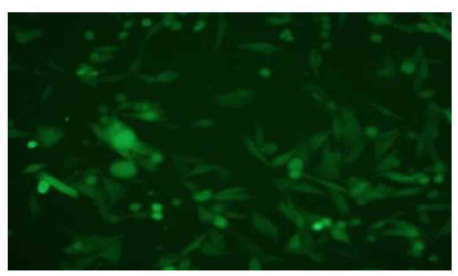

HCC1937/shDTX1

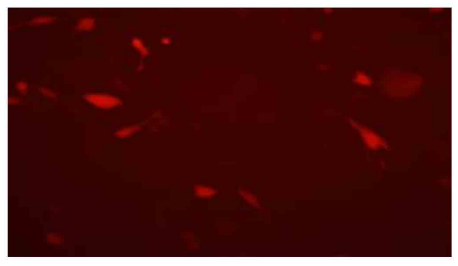

BT474/DTX1
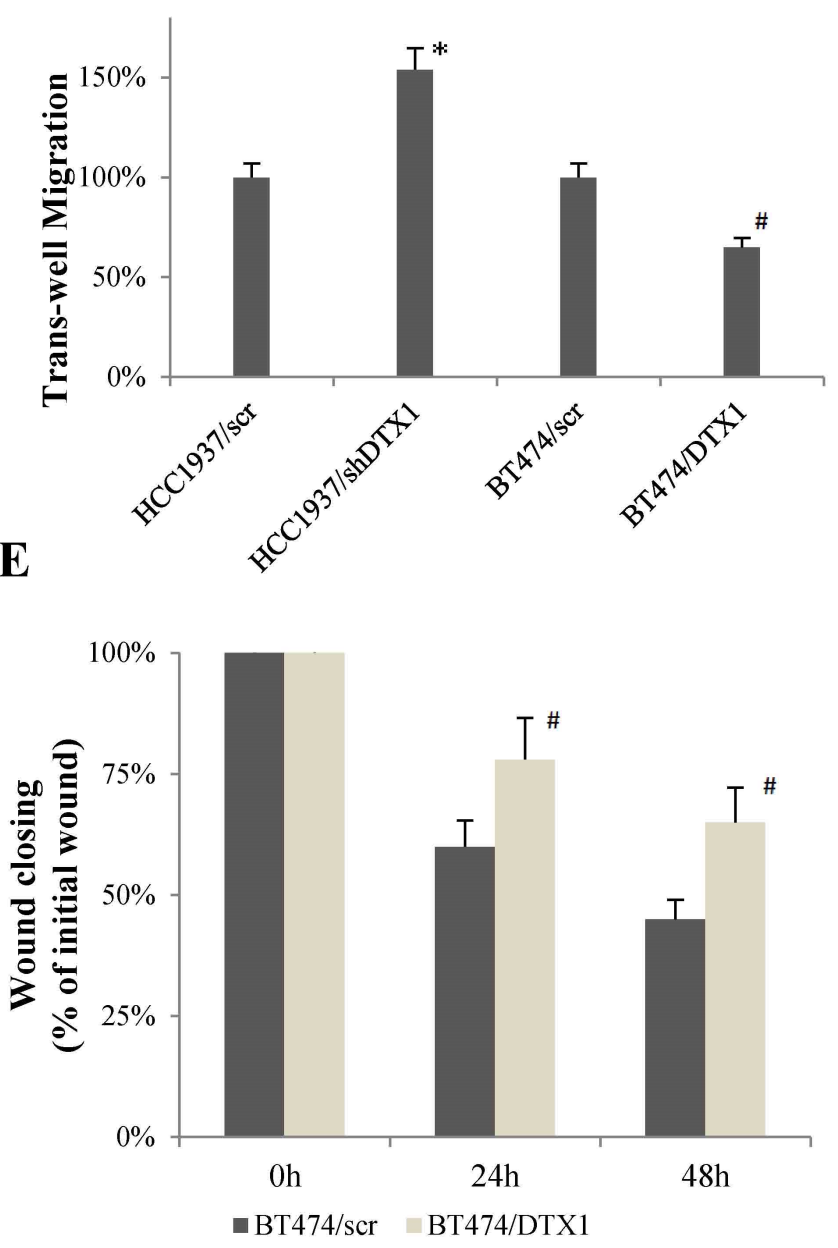

\section{Figure 2}

Lower DTX1 promoted breast cancer cell proliferation, migration and invasion. A: mRNA expression of DTX1 in different cell lines. B: HCC1937 and BT474 cell lines for DTX1 knockdown and overexpression studies. C: The growth and migration of HCC1937/ shDTX1cells was higher while BT474/DTX1 cells was lower compared to control cells ( ${ }^{*}<0.01, \# P<0.01$ ). D: Wound closing was massively accelerated in 
HCC1937/shDTX1 cells compared to controls ( ${ }^{*} \mathrm{P}<0.01$ ). E: Wound closing was significantly reduced in BT474/DTX1 cells $(\# p<0.05)$.

A

$$
\begin{array}{cccc}
\text { HCC1937 } & \text { HCC1937 } & \text { BT474 } & \text { BT474 } \\
\text { /scr } & \text { /shDTX1 } & \text { /scr } & \text { /DTX1 }
\end{array}
$$
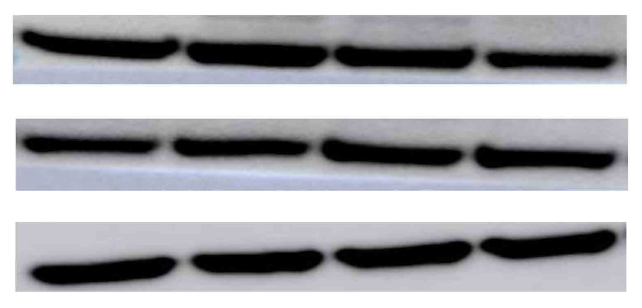

Jagged1

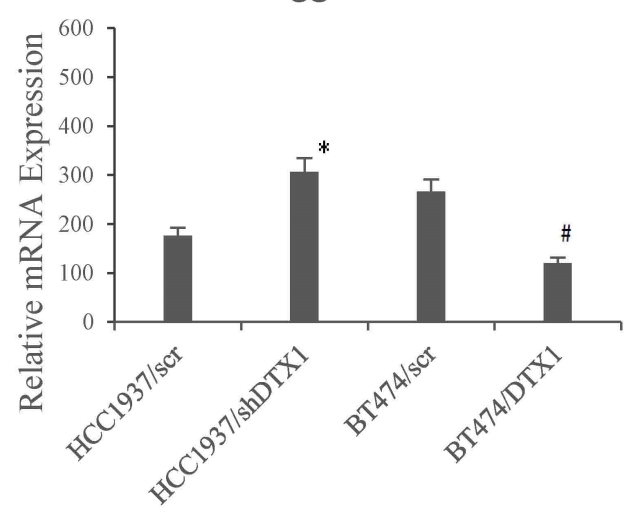

B

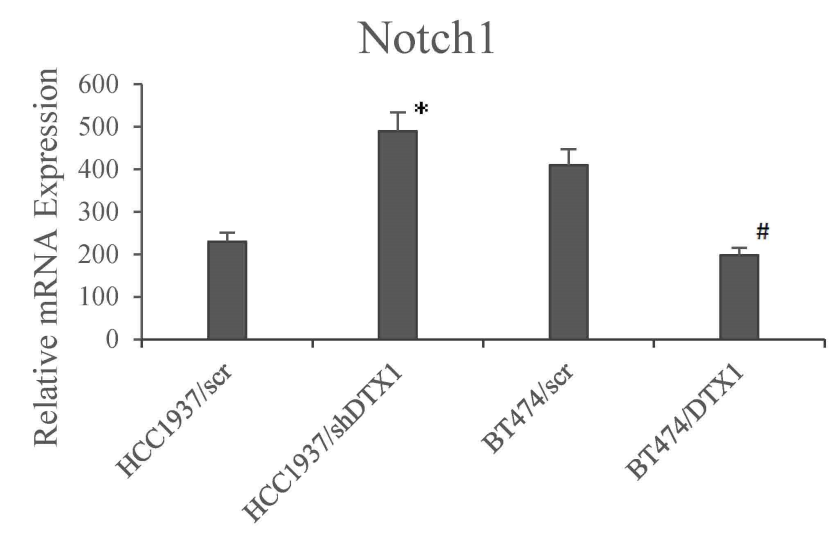

HES1

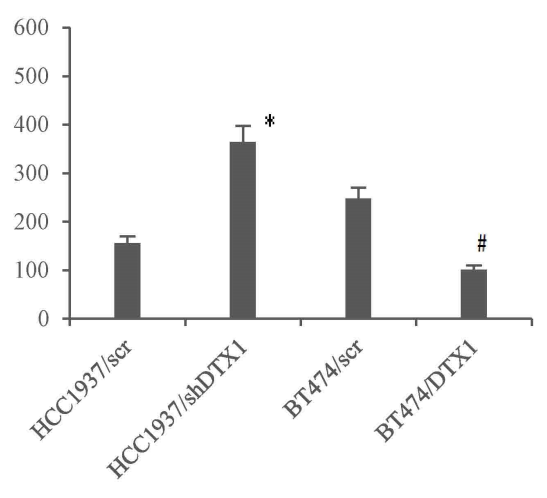

DTX1

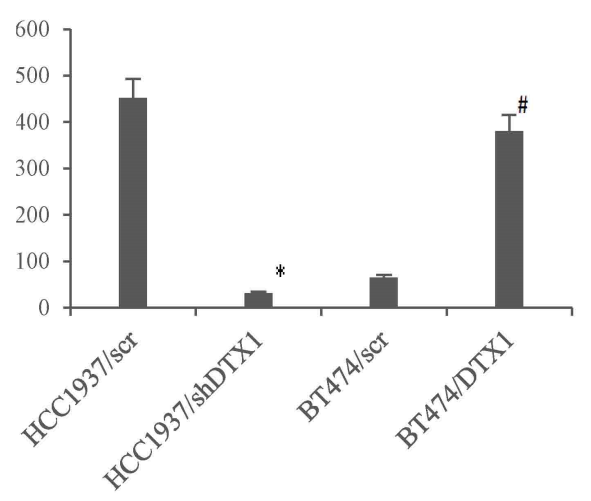

\section{Figure 3}

DTX1 promoted cell proliferation and migration via NOTCH/DTX1/ PI3K/AKT pathways. A: The expression of PI3K/AKT signal molecules in HCC1937/ shDTX1 cells, BT474/DTX1 cells and control cells by Western Blot, full-length blots were presented in Supplementary Figure 1. B: The mRNA expression of Notch1, Jagged1, HES1 and DTX1 in HCC1937/ shDTX1 cells, BT474/DTX1 cells and control cells by RTPCR (compared with respective control cells, * $\mathrm{P}<0.01$, \# $\mathrm{P}<0.01$ ). 
A

B
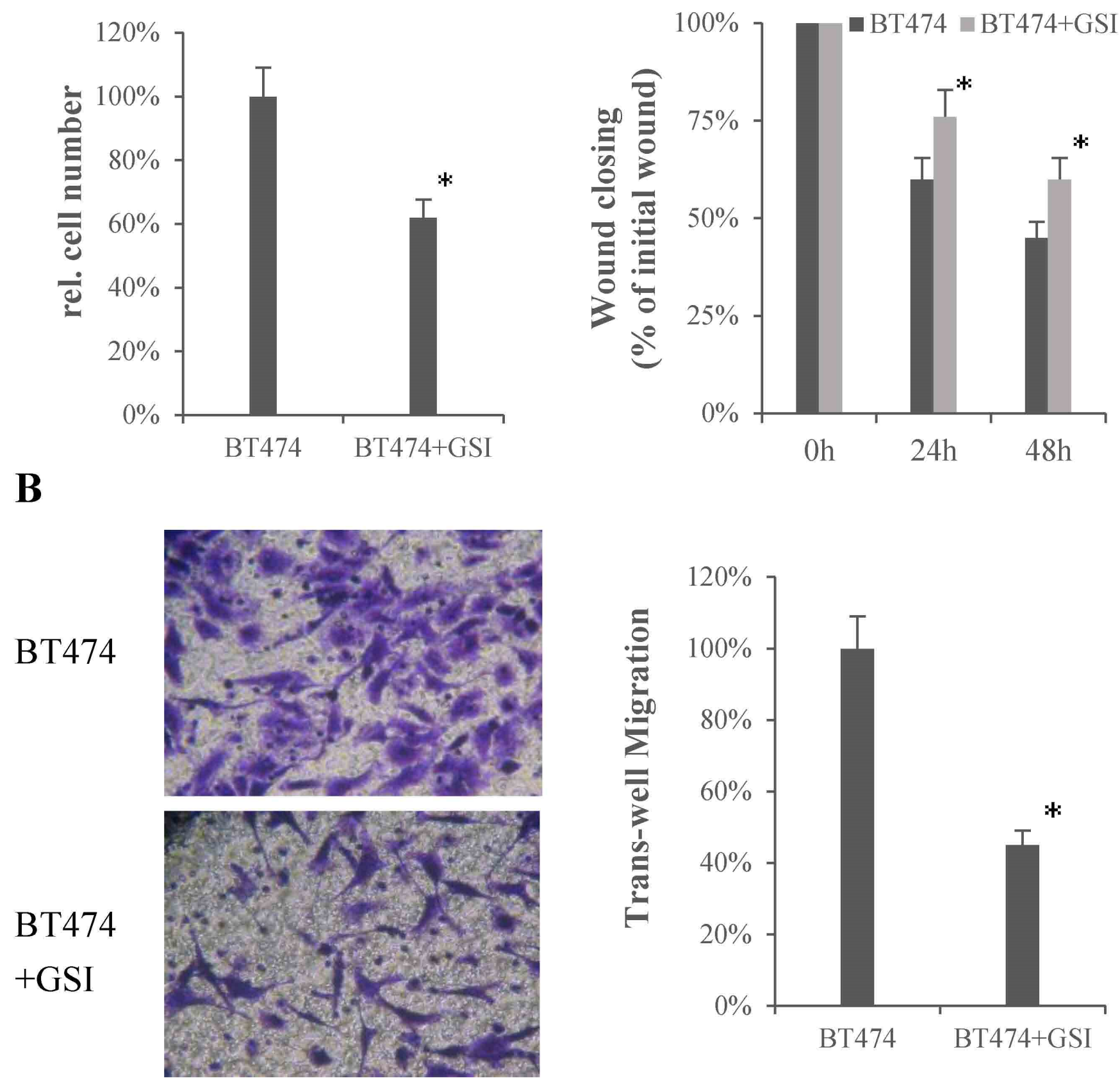

Figure 4

GSI inhibited proliferation, migration and invasion of BT474 cells. A: After GSI treatment, the proliferation and invasion of BT474 cells significantly decreased $\left({ }^{*} P<0.01\right)$. B: The migration and invasion of $B T 474$ cells significantly decreased ( $\left.{ }^{\star} P<0.01\right)$.

\section{Supplementary Files}


This is a list of supplementary files associated with this preprint. Click to download.

- table1.docx

- table3.docx

- supplementarymaterial.docx

- table2.docx 